\title{
Heliostat field aiming strategies for solar central receivers
}

\section{Estrategias de apuntamiento de campos de heliostatos en receptores solares centrales}

\author{
Alberto Sánchez-González \\ ISE Research Group, Department of thermal and fluids engineering, Universidad Carlos III de Madrid, \\ Av. Universidad 30, 28911 Leganés, Madrid, Spain \\ (*) E-mail: asgonzal@ing.uc3m.es \\ S: SEDOPTICA member \\ Received / Recibido: 21/06/2019 Accepted / Aceptado: 26/11/2019 \\ DOI: $10.7149 /$ OPA.52.4.51024
}

\begin{abstract}
:
This thesis work deals with the development and validation of four optical models for Solar Power Tower technology. The first model accurately computes the solar flux distribution incident on any central receiver reflected by a single heliostat; the novelty relies on the oblique projection of the receiver mesh onto the image plane where an analytic convolution function is evaluated. The second model determines the canting errors in the facets of real heliostats using a deterministic optimization algorithm that minimizes the difference between computed flux maps and experimental images captured on a Lambertian target. The third model extends the basic model to complete fields of heliostats where a symmetric aiming strategy is developed; just a single parameter, novel $k$ factor, is needed to aim all the heliostats. The last aiming model maximizes the output in molten salt receivers while meeting the corrosion and thermal stress constraints; which are translated into allowable flux densities.
\end{abstract}

Key words: Concentrating solar power tower; Central receiver system; Flux density distribution; Multi-aiming strategy; Heliostat optical quality.

\section{RESUMEN:}

Este trabajo de doctorado trata del desarrollo y validación de cuatro modelos ópticos para la tecnología de plantas solares de torre. El primer modelo calcula con precisión la distribución de flujo solar incidente en receptores centrales reflejada por un heliostato; la novedad radica en la proyección oblicua de la malla de cálculo desde el receptor hasta el plano imagen donde se evalúa una función analítica de convolución. El segundo modelo determina los errores de canteo en las facetas de heliostatos reales mediante un algoritmo determinista que minimiza la diferencia entre mapas de flujo simulados e imágenes experimentales tomadas en un blanco lambertiano. El tercer modelo extiende el modelo básico a campos completos de heliostatos siguiendo un apuntamiento simétrico; con un único parámetro, llamado factor $k$, se apuntan todos los heliostatos. El último modelo de apuntamiento maximiza la energía térmica obtenida en receptores de sales fundidas a la vez que respeta las limitaciones de corrosión y stress térmico; las cuales son traducidas en densidades de flujo permisibles.

Palabras clave: Torre solar de concentración; Sistema de receptor central; Distribución de flujo; estrategia de apuntamiento; Calidad óptica de heliostatos.

\section{REFERENCES AND LINKS}

[1] K. Lovegrove and W. Stein, Concentrating solar power technology: principles, developments and applications. Elsevier (2012). 
[2] L. L. Vant-Hull, "Central tower concentrating solar power (CSP) systems" in Concentrating solar power technology: principles, developments and applications. Woodhead Publish.-Elsevier (2012).

[3] P. Garcia, A. Ferrière, and J.-J. Bezian, "Codes for solar flux calculation dedicated to central receiver system applications: A comparative review," Sol. Energy 82, 189-197 (2008).

[4] A. Sánchez-González, "Heliostat field aiming strategies for solar central receivers," Universidad Carlos III de Madrid, Doctoral thesis (2016). https://e-archivo.uc3m.es/handle/10016/24712

[5] A. Sánchez-González and D. Santana, "Solar flux distribution on central receivers: A projection method from analytic function," Renew. Energy 74, 576-587 (2015).

[6] F. J. Collado, A. Gómez, and J. A. Turégano, "An analytic function for the flux density due to sunlight reflected from a heliostat," Sol. Energy 37, 215-234 (1986).

[7] T. Wendelin, "SolTRACE: a new optical modeling tool for concentrating solar optics," in ISEC (2003).

[8] S. A. Jones, "A comparison of on-axis and off-axis heliostat alignment strategies," in American Solar Energy Society Conference, 95-100 (1996).

[9] A. Sánchez-González, C. Caliot, A. Ferriere, and D. Santana, "Determination of heliostat canting errors via deterministic optimization," Sol. Energy 150, 136-146 (2017).

[10] S. Theodoridis and K. Koutroumbas, "Template Matching," in Pattern Recognition, Academic press - Elsevier, 481-519 (2009).

[11] J. S. Arora, “Global Optimization Concepts and Methods," in Introduction to Optimum Design, Elsevier, 681-712 (2012).

[12] D. R. Jones, C. D. Perttunen, and B. E. Stuckman, "Lipschitzian optimization without the Lipschitz constant," J. Optim. Theory Appl. 79, 157-181 (1993).

[13] D. E. Finkel, “DIRECT Optimization Algorithm User Guide,” (2003).

[14] A. Sánchez-González, M. R. Rodríguez-Sánchez, and D. Santana, "Aiming factor to flatten the flux distribution on cylindrical receivers," Energy 153, 113-125 (2018).

[15] G. Sassi, "Some notes on shadow and blockage effects," Sol. Energy 31, 331-333 (1983).

[16] L. L. Vant-Hull, "The Role of 'Allowable Flux Density' in the Design and Operation of Molten-Salt Solar Central Receivers," J. Sol. Energy Eng. 124, 165-169 (2002).

[17] A. Sánchez-González"FluxSPT" Software tool (2018) https://ise.uc3m.es/research/solar-energy/fluxspt

[18] A. Salomé, F. Chhel, G. Flamant, A. Ferrière, and F. Thiery, "Control of the flux distribution on a solar tower receiver using an optimized aiming point strategy: Application to THEMIS solar tower," Sol. Energy 94, 352-366 (2013).

[19] S. M. Besarati, D. Yogi Goswami, and E. K. Stefanakos, "Optimal heliostat aiming strategy for uniform distribution of heat flux on the receiver of a solar power tower plant," Energy Convers. Manag. 84, 234-243 (2014).

[20] B. Belhomme, R. Pitz-Paal, and P. Schwarzbözl, “Optimization of Heliostat Aim Point Selection for Central Receiver Systems Based on the Ant Colony Optimization Metaheuristic," J. Sol. Energy Eng. 136, 11005-1-7 (2013).

[21] F. J. García-Martín, M. Berenguel, A. Valverde, and E. F. Camacho, "Heuristic knowledge-based heliostat field control for the optimization of the temperature distribution in a volumetric receiver," Sol. Energy 66, 355-369 (1999).

[22] M. R. Rodríguez-Sánchez, A. Soria-Verdugo, J. A. Almendros-Ibáñez, A. Acosta-Iborra, and D. Santana, "Thermal design guidelines of solar power towers," Appl. Therm. Eng. 63, 428-438 (2014).

[23] M. R. Rodríguez-Sánchez, C. Marugan-Cruz, A. Acosta-Iborra, and D. Santana, "Comparison of simplified heat transfer models and CFD simulations for molten salt external receiver," Appl. Therm. Eng. 73, 991-1003 (2014).

[24] A. Sánchez-González, M. R. Rodríguez-Sánchez, and D. Santana, “Aiming strategy model based on allowable flux densities for molten salt central receivers,” Sol. Energy 157, 1130-1144 (2017). 


\section{Introduction}

Concentrating Solar Power (CSP) is one of the clean technologies intended to gradually replace nonrenewable energy sources. In CSP systems, solar beam radiation is reflected and concentrated by an array of mirrors, and the final output is usually electricity. Depending on the way in which solar radiation is concentrated, CSP is classified into four different systems [1]: parabolic trough, linear Fresnel reflector, parabolic dish and solar power tower.

This doctoral research is specifically devoted to Solar Power Tower (SPT) systems. A SPT plant consist of a field of tacking mirrors, named heliostats, that concentrate direct solar radiation into a central receiver on the top of a tower [2]. A heat transfer fluid is heated by the concentrated radiation throughout such thermal receiver. The thermal energy, which may be stored as hot molten salt in tanks, is finally converted into mechanical energy, and eventually electricity, by means of a thermodynamic, generally Rankine, cycle. A SPT plant thus consists of four systems: heliostat field, central receiver, thermal storage and power block; as depicted in Fig. 1.

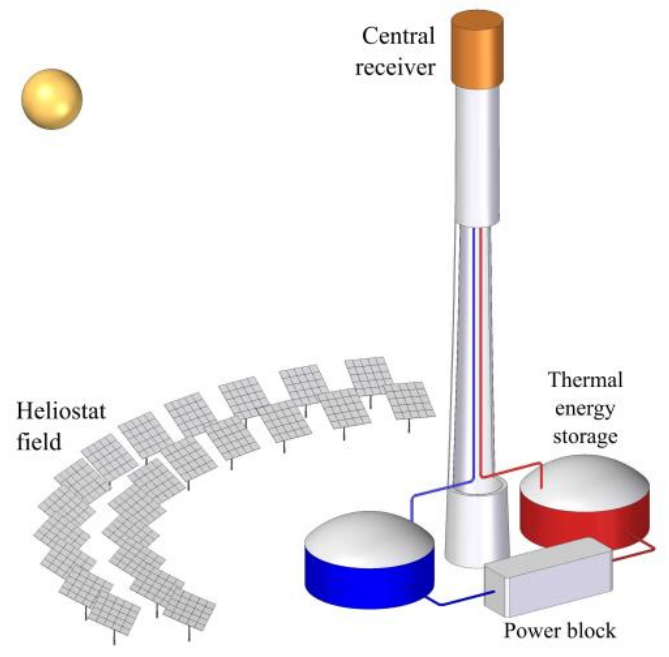

Fig. 1. SPT plant.

Deployment and consolidation of SPT technology demand models and tools to assist in design and operation phases. This thesis essentially deals with the development of accurate and fast optical models to analyze the interaction between heliostat field and central receiver. For the computation of the flux distribution concentrated on the receiver two approaches can be identified [3]: Monte Carlo Ray Tracing, an statistical method that traces a bundle of random rays; and convolution, that superposes the mathematical functions of solar distribution (or sunshape), concentration and optical errors. Because of its fast computation and suitability for optimization studies, this work is grounded on the convolution approach, providing enhancements to existing analytic functions.

The Ph.D. dissertation [4], on which this paper is based, provides new contributions to the optical modelling, flux mapping and aiming strategies for solar central receivers. Four computational models have been developed, in the achievement of the four key objectives of the thesis:

1. Build and validate an optical model to map the flux incident on central receivers, both flat and cylindrical. Model requirements are accuracy and computation speed.

2. Develop and validate a methodology to determine optical errors (canting) from heliostat flux images measured on a target. Optimization techniques are used to find out canting errors.

3. Carry out an aiming strategy model for complete heliostat fields to generate different patterns of flux maps, including flat distribution. A single parameter connected to the spillage losses is pursued.

4. Develop a heliostat field aiming strategy for molten salt cylindrical receivers to maximize receiver thermal output, while carefully meeting corrosion and thermal stress limits. 
The following sections subsequently present and describe each optical model.

\section{Projection method for flux distribution from a single heliostat}

The first model, on which the rest of the models are built up, computes the flux density distribution incident on any kind of central receiver caused by a single heliostat (i.e. tracking mirror). Since analytic functions derived from the convolution approach are conveniently defined on the image plane, a projection methodology is proposed to accurately compute the flux distribution. This procedure solves the distorted spot found in actual receivers, when the incidence angle on the receiver increases [5].

The oblique projection is accomplished with a four-step methodology, as illustrated in Fig. 2: 1) Receiver panels are discretized in a mesh of equally spaced nodes; 2 ) Mesh nodes are projected onto the image plane in the direction of the central reflected ray t; 3 ) The analytic function is evaluated at the nodes in the image plane; and 4) Flux density at images nodes is multiplied by the cosine of the incidence angle on the receiver.
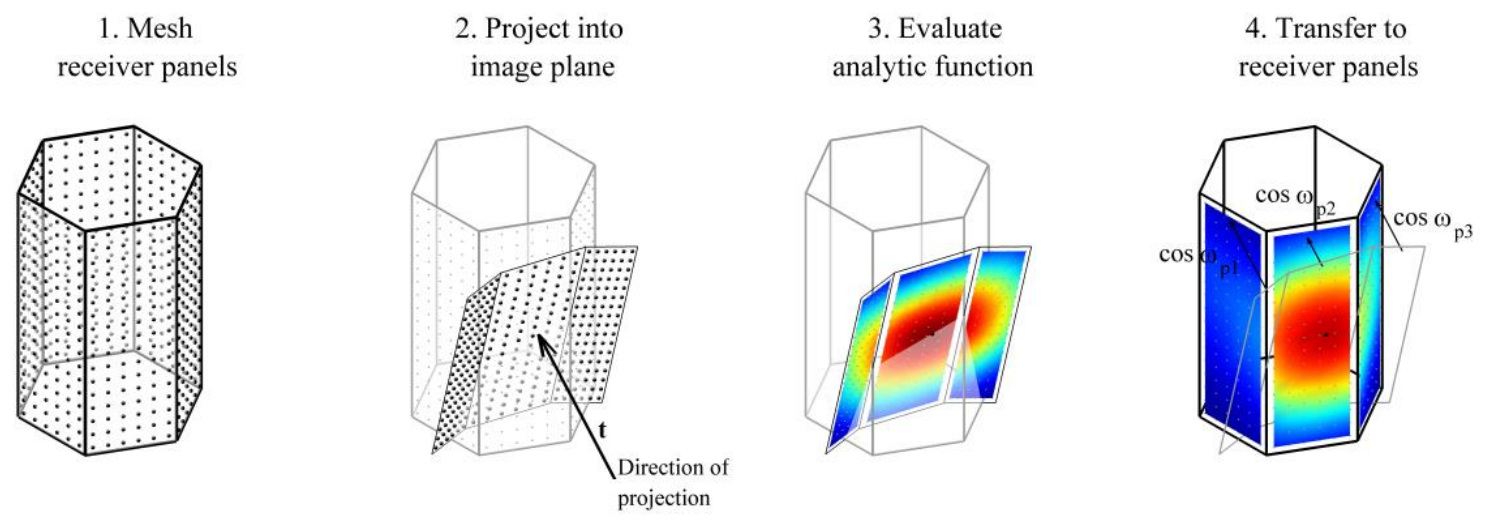

Fig. 2. Projection methodology.

A proper selection of the systems of coordinates, Fig. 3 (a), is essential to the success of all the transformations taking place. The most important one is the transformation from target (st) to image (si) system of coordinates, Fig. 3 (b). This transformation involves two rotations about the azimuth and elevation angles, respectively $\alpha_{t}$ and $\varepsilon_{t}$. In matrix notation, such a transformation is developed through the following Equation:

$$
\left[\begin{array}{c}
x \\
y \\
z
\end{array}\right]_{s i}=\left[\begin{array}{ccc}
\cos \alpha_{t} & 0 & \sin \alpha_{t} \\
-\sin \alpha_{t} \cdot \sin \varepsilon_{t} & \cos \varepsilon_{t} & \cos \alpha_{t} \cdot \sin \varepsilon_{t} \\
-\sin \alpha_{t} \cdot \cos \varepsilon_{t} & -\sin \varepsilon_{t} & \cos \alpha_{t} \cdot \cos \varepsilon_{t}
\end{array}\right] \cdot\left[\begin{array}{c}
x \\
y \\
z
\end{array}\right]_{s t}
$$
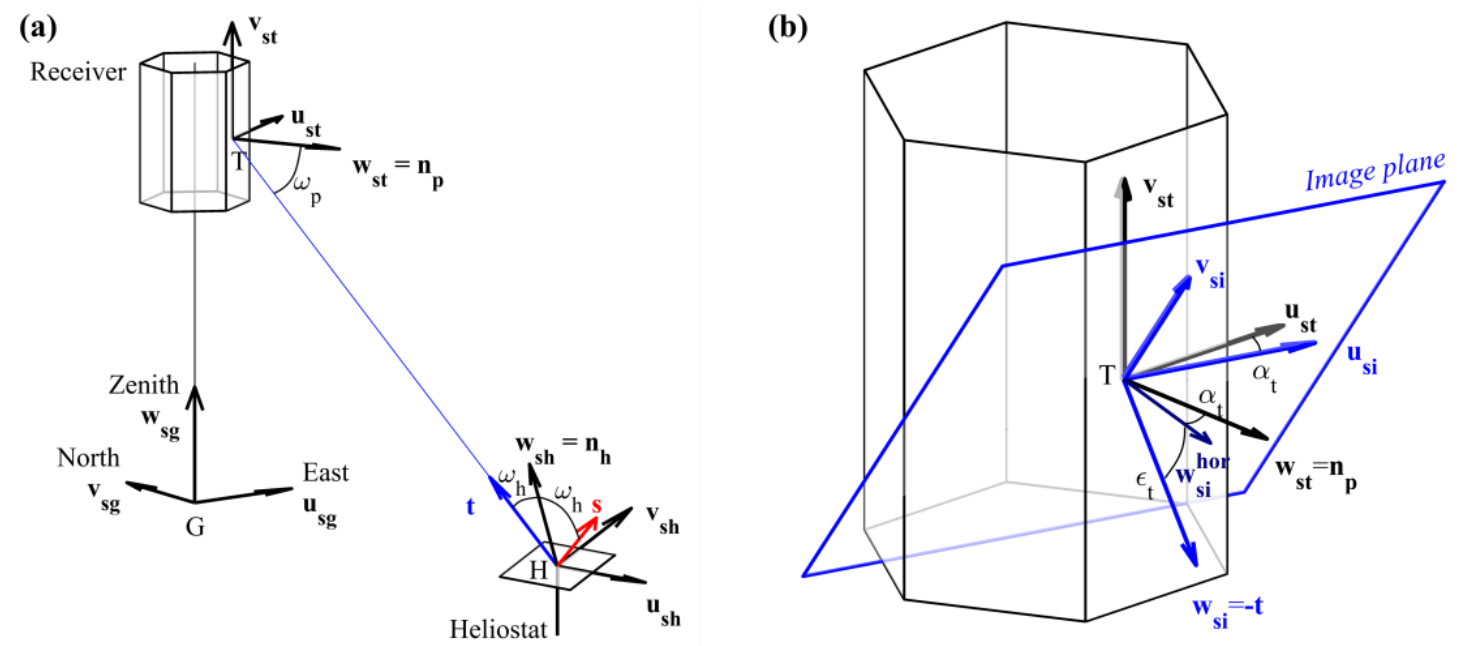

Fig. 3. Systems of coordinates: (a) global, heliostat, and target; (b) transformation from target to image coordinate system. 
Once in the image coordinate system, the projection of the receiver nodes onto the image plane (i.e. step 2) is straightforward. While $x$ and $y$ coordinates remain fixed, the $z$ coordinate drops to zero, thus reaching the image plane. Symbolizing the projection onto the image plane with superscript image, the projection is algebraically equivalent to:

$$
x_{s i}^{\text {image }}=x_{s i}, \quad y_{s i}^{\text {image }}=y_{s i}, \quad z_{s i}^{\text {image }}=0
$$

Existing analytic functions on the image plane rely on the gaussian distribution of the reflected beam. Because of its accuracy describing the flux distribution due to rectangular focusing heliostats, the UNIZAR function on the image plane by Collado et. al [6] is taken in the dissertation, specifically for step 3. In terms of concentration ratio, $\mathrm{C}$, the distribution on any receiver node $[i, j]$ belonging to panel $p$ satisfies Equation (3), as for step 4. The methodology is also advantageous for the calculation of the spillage losses, or its reciprocal the intercept factor, fint, as defined in the following Equation.

$$
\begin{array}{r}
C_{i, j, p}=C_{i, j, p}^{\text {image }} \cdot \cos \omega_{p} \\
f_{\text {int }}=\frac{\sum_{i, j, p} C_{i, j, p} \cdot \Delta x \cdot \Delta y}{\cos \omega_{h} \cdot A M}
\end{array}
$$

The proposed model has been validated for both flat and cylindrical receivers. For a given heliostat at Plataforma Solar de Almería, Fig. 4 contrasts the model output (continuous lines) to both experimental measurements (a), and Ray Tracing simulation (b) on a flat lambertian target. As can be seen, the model reproduces the distorted spot found at a high incidence angle $\left(26.5^{\circ}\right)$. Compared to the experimental image Fig. 4 (a), the general pattern is followed while particular deviations are encountered. Such slight differences can be explained by: misalignment of the heliostat, its elastic deformation, as well as experimental uncertainty in the measurements.

(a)

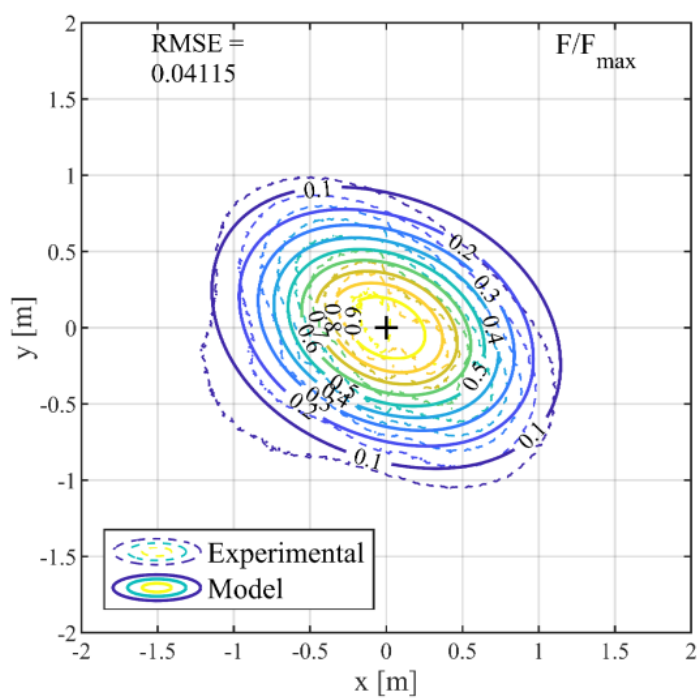

(b)

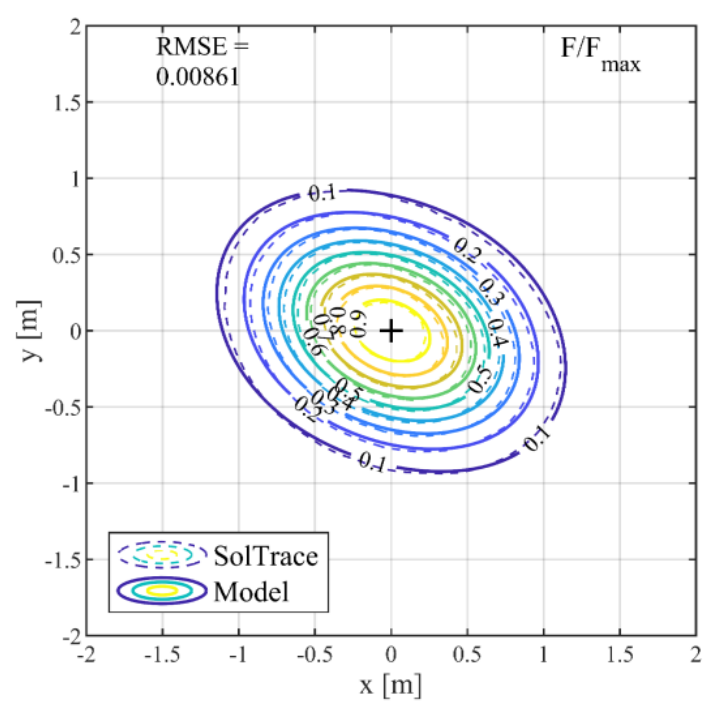

Fig. 4. Contours of normalized flux by heliostat H61 at Plat. Solar de Almería. Comparison with measurement (a) and SolTrace (b).

Compared to Ray Tracing simulations, Fig. 4 (b), in SolTrace [7], the proposed code underestimates receiver interception up to $2 \%$. Our model takes 50 times less computation time with even higher level of resolution.

\section{Determination of optical canting errors in heliostats}

Optical beam quality of heliostats depends on its correct alignment, which involves two operations [8]: mirror focusing (i.e. slightly bending the mirror surface to minimize the reflected sun image) and heliostat canting (i.e. tilting the mirror modules to aim at the same point). Misalignment of mirror facets leads to heliostats with poor optical quality, such as multiple spots and/or large beam sizes.

This section presents a novel methodology to find out canting errors in the mirror modules of real heliostats [9]. An optimization procedure has been established to fit simulated flux distributions from heliostats to 
those measured on a lambertian target. In this instance, a large series of raw experimental images was taken at THEMIS solar plant, belonging to PROMES-CNRS laboratory.

Because of the absence of accurate measurements on the heliostat orientation at THEMIS, an inference procedure was firstly set. The 3-step process to infer the instantaneous heliostat aim point on the target planes, as graphically depicted in Fig. 6, consists on:

1. Calculate the centroid of the experimental image, WCexp.

2. With target point in WCexp, simulate the flux distribution and calculate its centroid, WC mod.

3. Shift the target point to WCexp-WCmod.

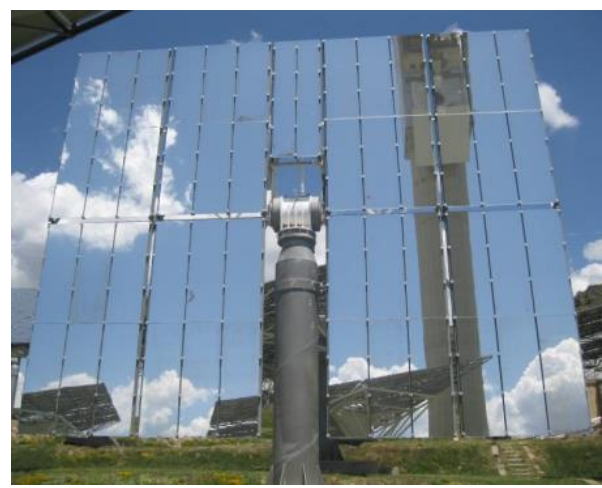

Fig. 5. Heliostat B10 at THEMIS solar plant.
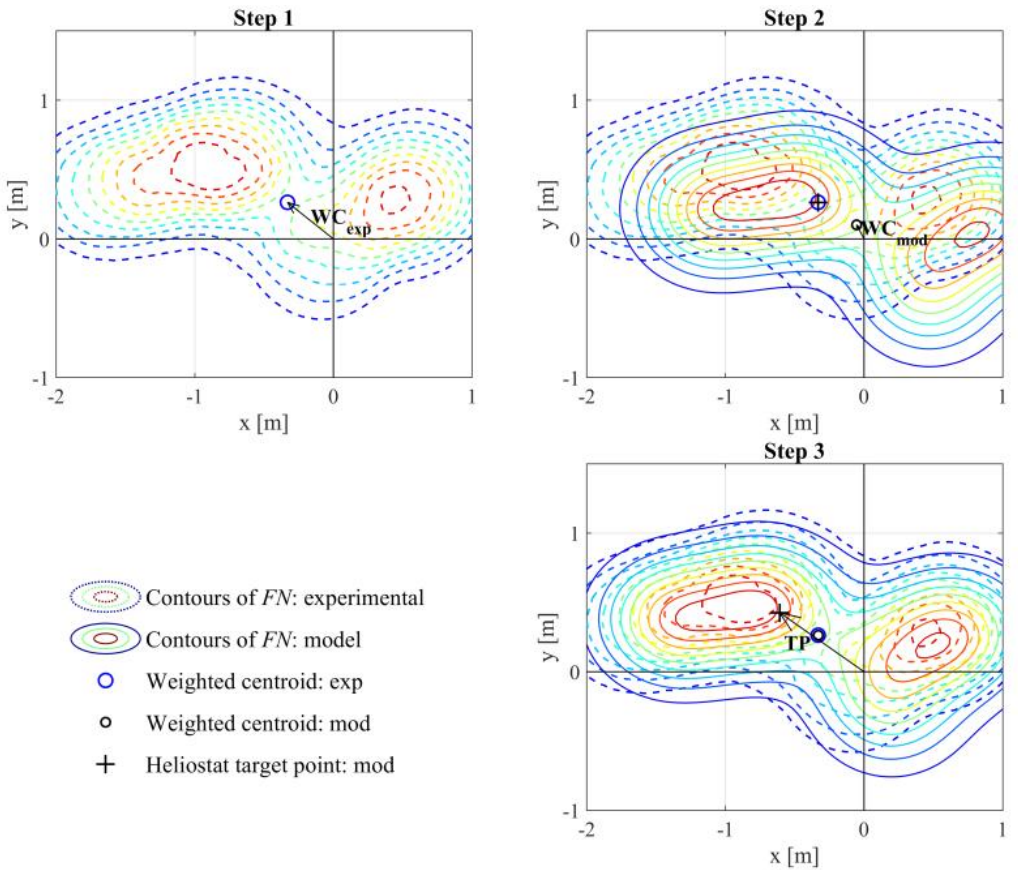

Fig. 6. Inference of heliostat target point.

This procedure minimizes the difference between captured images and computed flux maps, taking advantage of the model described in the previous section [5]. For CETHEL heliostats with 9 mirror modules (Fig.5), there are a total of 17 degrees of freedom (i.e. angular errors, $\delta_{x}$ and $\delta_{y}$ ). This is an optimization problem, where the cross-correlation coefficient (CCC), also known as Pearson correlation coefficient, parameter frequently used in pattern recognition [10], was finally adopted.

Among the plethora of global optimization methods [11], the solution failed under metaheuristic algorithms. Because of its deterministic approach and reliability, it was adopted the Dividing RECTangles 
(DIRECT) algorithm, that divides the search space into smaller hypercubes to find the global minimum [12]. In this study, the Matlab implementation by Finkel [13] was utilized. The optimization procedure was applied to three heliostat at THEMIS, taking less than 2 hours of computation time to reach a convergent solution for each of them.

The validity of the methodology was assessed by in situ readjusting the heliostat with lowest optical quality, on the basis of the optimization results. For that selected heliostat, Fig. 7 represents the measured flux distributions before (left) and after (right) adjustment. The fitting between original experimental image (continuous contours) and optimization result (dashed) is shown in the middle of the Figure, where the correlation coefficient nearly reaches $97 \%$.

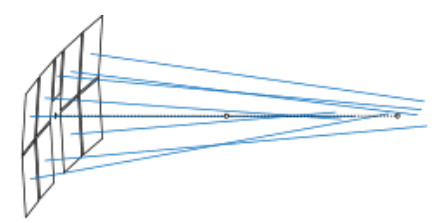

Before readjustment (exp)

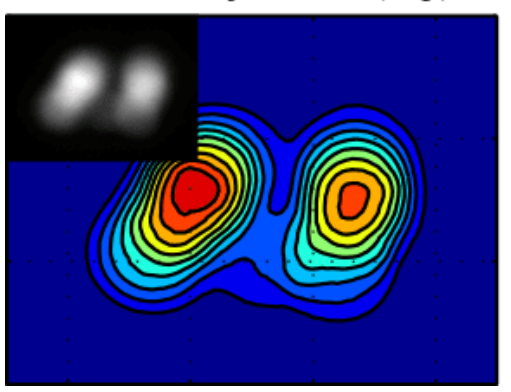

Fig. 7. Graphical summary on the determination of canting errors and heliostat readjustment.
Canting errors from experimental heliostat images

\section{DIRECT algorithm}

applied to optical model

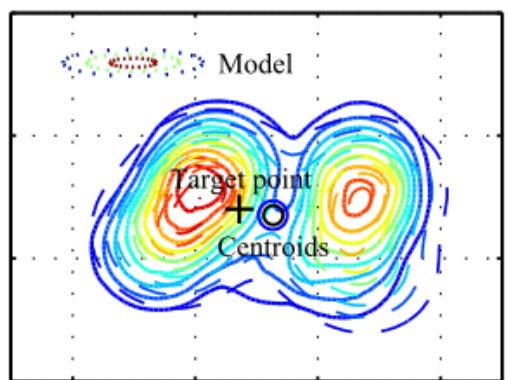

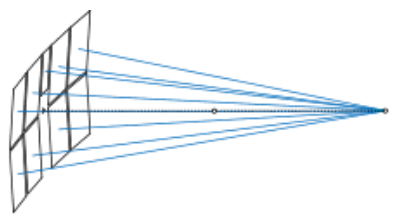

After readjustment (exp)

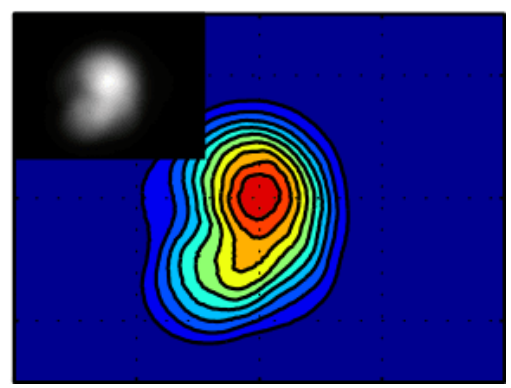

\section{Symmetric flux maps from a field of heliostats: aiming factor approach}

The base model in section 2 was extended to map the flux distribution on a cylindrical receiver caused by a surrounding field of heliostats. The benefit of cylindrical compared to flat receivers is related with the capacity of receiving concentrated solar radiation from a whole surrounding heliostat field $\left(360^{\circ}\right)$. This way, cylindrical receivers promote higher temperatures of the heat transfer fluid at its outlet.

This extended model superimposes the flux map generated by every single heliostat in a surrounding field [14]. All sources of optical losses, as stated in the heliostat optical efficiency (Eq. (5)), were taken into account: cosine $(\cos \omega h)$, reflectivity $(\rho)$, atmospheric attenuation $\left(f_{a t}\right)$, spillage/interception $\left(f_{\text {int }}\right)$, and shading and blocking $\left(f_{s b}\right)$. Special attention was paid to the computation of shading and blocking losses, by means of parallel projection of neighbouring heliostats [15].

\section{4.a. Symmetric flux maps and aiming factor, $k$}

$$
\eta_{h}=\cos \omega_{h} \cdot \rho \cdot f_{s b} \cdot f_{a t} \cdot f_{\text {int }}
$$

Single equatorial aiming leads to hot spots and thermal stresses in the receiver, which have to be avoided to extend its lifetime. In order to procure nearly symmetric flux maps about the receiver equatorial plane, Vant-Hull [16] suggested to alternatively aim each row of heliostats to the top and to the bottom half of the receiver. Herein, even rows of heliostats aim tangentially to the lower edge, while odd rows to the upper edge, as displayed in Fig. 8 (right).

The beam reflected by a heliostat onto the receiver does not have a defined radius (i.e. size), because of its inherently gaussian nature. By analogy with the circular normal distribution, 68\%, 95\% and 99.7\% of the total flux is within a cone of respective aperture angle $\sigma_{\mathrm{e}}, 2 \sigma_{\mathrm{e}}$ and $3 \sigma_{\mathrm{e}}$, being $\sigma_{\mathrm{e}}$ the effective standard deviation. Thus, it is defined a $k$ parameter -named aiming factor-, typically ranging between 0 and 3 , so that the radius of the beam on the image plane $B R_{s i}$, depending on the heliostat-to-target distance (slant range, $S L R$ ), can be estimated with Eq. (6). And, known the elevation angle of the target vector ( $\varepsilon t$ ), the beam radius on the receiver vertical follows Eq. (7). 


$$
\begin{gathered}
\text { ÓPTICA PURA Y APLICADA } \\
\text { www.sedoptica.es } \\
B R_{k}^{S i}=S L R \cdot \tan \left(k \cdot \sigma_{e}\right) \simeq k \cdot S L R \cdot \sigma_{e} \\
B R_{k}=k \frac{S L R \cdot \sigma_{e}}{\cos \varepsilon_{t}}
\end{gathered}
$$

Finally, given a $k$ value, the heliostat is pointed at a distance $B R k$ from the upper (odd rows) or lower (even rows) receiver edge. The targeting process is depicted in the left of Fig. 8. It can be easily deduced that the lower the value of $k$, the higher the spillage losses are; in such a way that $k$ factor is a parameter controlling both aiming and interception.

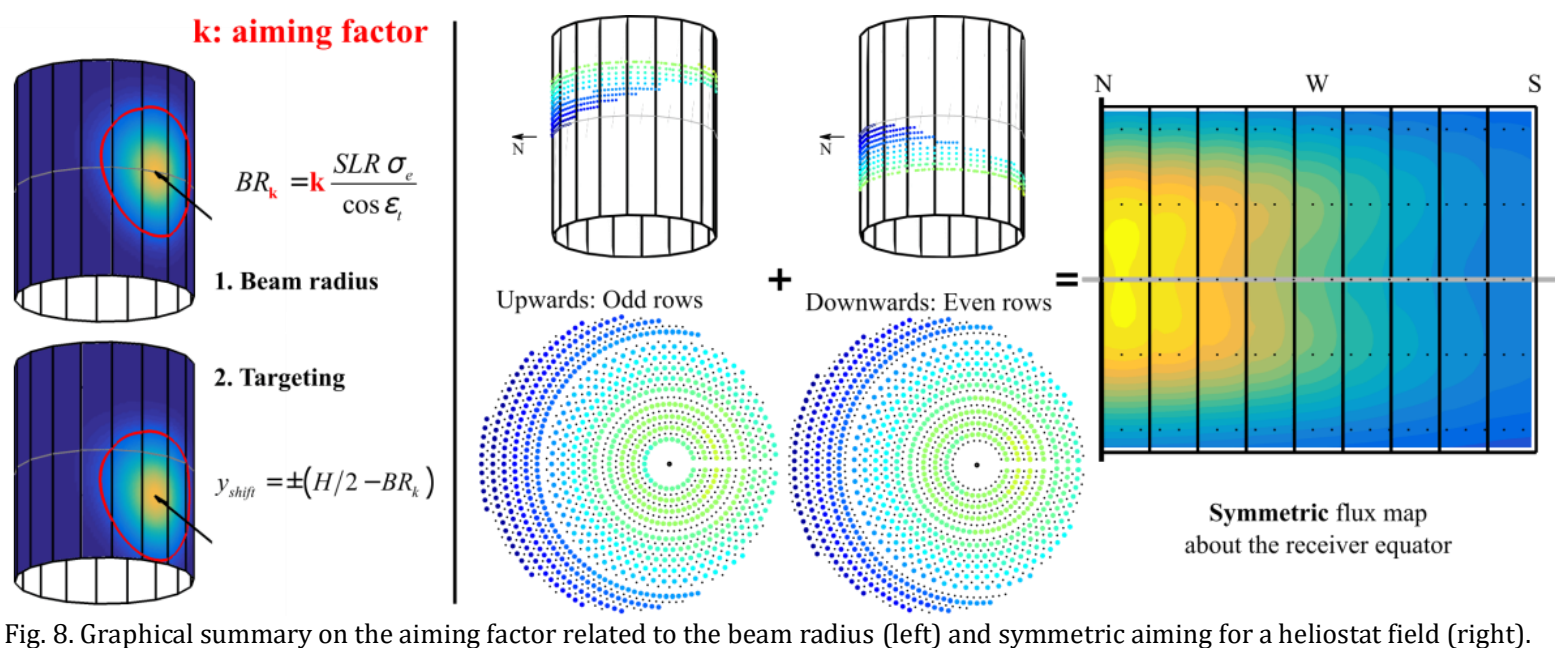

This symmetric aiming strategy based on the aiming factor is implemented in FluxSPT software tool, available for free download from the link in Ref. [17].

\section{4.b. Flat aiming factors, kflat}

There might be an aiming factor for each heliostat field sector, so that the flux distribution would be the flattest possible. By running a sweep algorithm, such $k$ flat factors for each sector are found. For the Dunhuang SPT case study at equinox 8:00, Fig. 9 shows the uniform flux map and vertical profiles for each receiver panel, arising from the kflat values. The corresponding aim point level for each heliostat is represented in Fig. 10 [14].
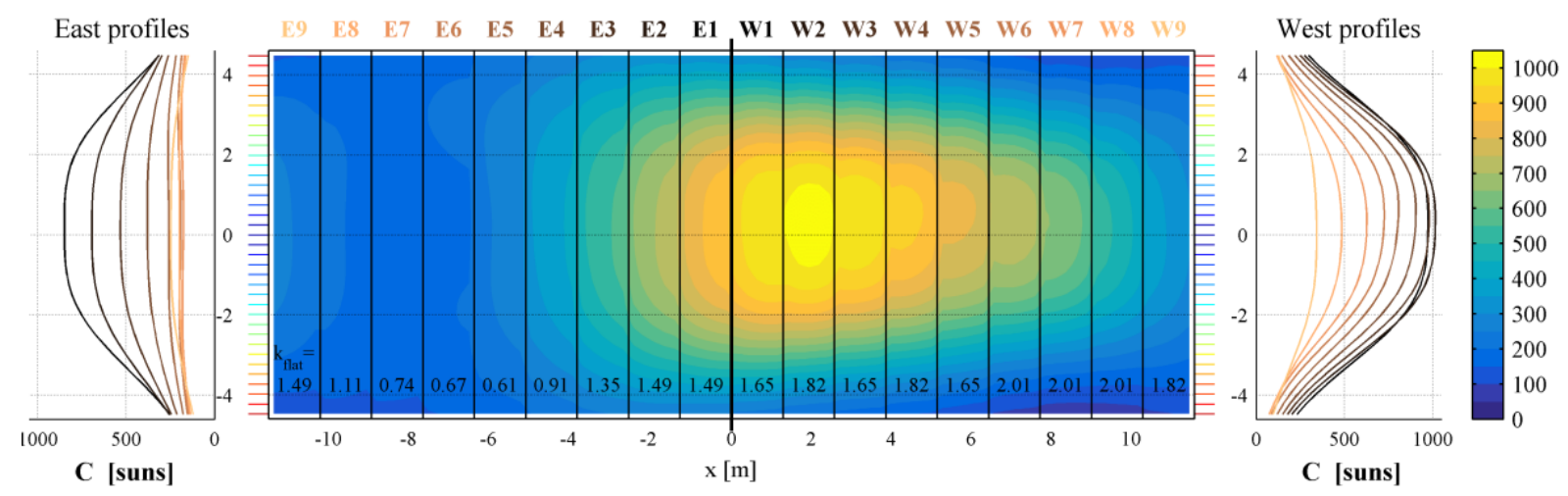

Fig. 9. Uniform flux map and flat profiles (east and west panels) using $k_{f l a t}$ factors. 


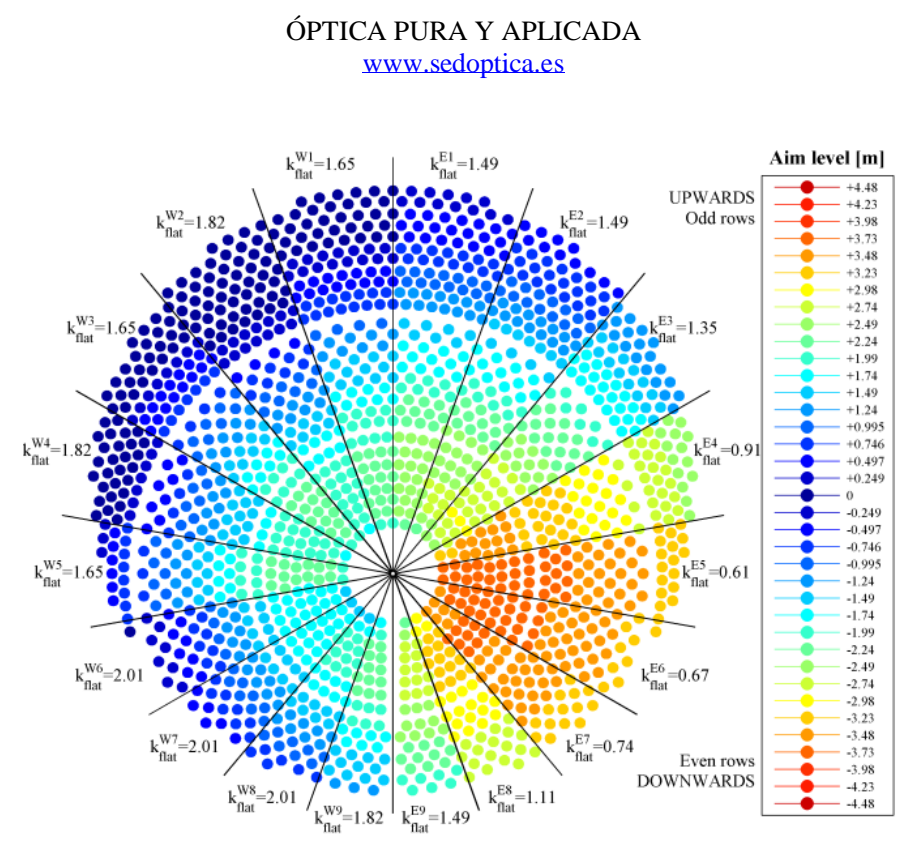

Fig. 10. Aim point level for each heliostat in Dunhuang field using $k$ fat factors.

\section{Aiming strategy for molten salt receivers: allowable flux density approach}

How to aim each and every heliostat in a field in order to maximize the receiver output and maintain its structural integrity is a key question for the reliable operation of SPT plants. Despite the importance, a number of studies have dealt with the aiming issue, e.g. [18-21]. In 2002, Vant-Hull introduced the concept of Allowable Flux Density, AFD, dependent on the heat transfer fluid temperature and flow rate [16].

On the basis of the concepts of AFD and aiming factor, as presented in previous section, a complete methodology to aim all the heliostats in a field was developed. This aiming strategy couples the previously presented optical model [5] and a thermal model for cylindrical receivers using molten salt as heat transfer fluid $[22,23]$. Limitations by corrosion (e.g. film temperature smaller than $630^{\circ} \mathrm{C}$ for tubes of alloy $800 \mathrm{H}$ ) and thermal stress (i.e. smaller than the ultimate tensile stress) are translated into AFDs, which can be handled by the optical model. From the receiver model, it was generated a database of AFD corr by corrosion and AFD strs by thermal stress, as shown in Fig. 11.

With inputs from the optical model and the database of AFDs, the aiming strategy model consists of two sequential algorithms, search and fit, as represented in the flowchart in Fig. 12. The global objective is to automatically aim the heliostats to collect as much as possible solar flux, while at the same time not exceeding the AFD limits [24].

The search algorithm finds the highest aiming factor for each field sector, ksector, with which the AFD limit is met. The symmetric aiming shown in previous section is also considered in the search algorithm, which in turn consists of two sequential routines: sweep and adjustment. Subsequently, the fit algorithm selects the heliostat target points to match the flux profile to the AFD limit. Regarding computation time in a standard PC, the search and fit algorithms takes respectively around 70 and $50 \mathrm{~s}$, then around two minutes in total; which is small compared to the feasible $43^{602}$ combinations in the case study. 

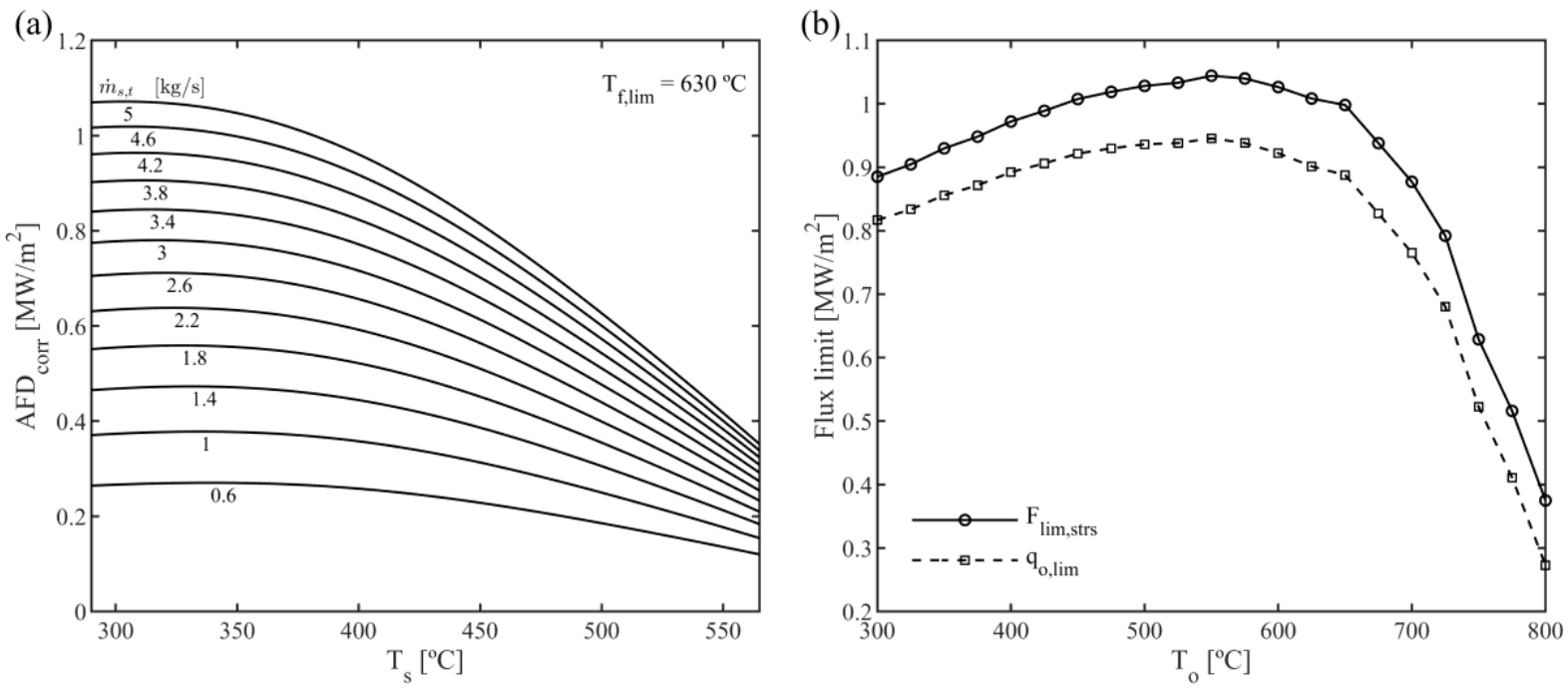

Fig. 11. (a) Allowable flux densities by receiver corrosion. (b) Flux limit by thermal stress.

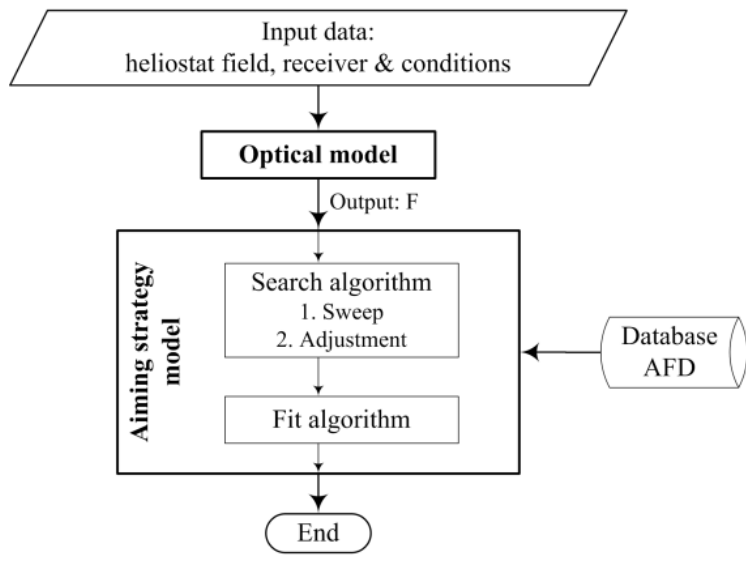

Fig. 12. Flowchart for the whole model.

For the Gemasolar SPT case study plant, whose heliostat field layout is shown in Fig. 13. Fig. 14 represents the result of the fitting process, where the flux profiles are smaller or matching the AFD limit set by the black thick line. The sequence of addition of each field row is also pointed out by the profiles colors, which are accommodated from lowest (colder colors) to highest (warmer colors) degrees of freedom in the vertical shifting. The bottom of Fig. 14 also represents the profiles of molten salt temperature (black) and film temperature (red), the latter not exceeding the corrosion limit of $630^{\circ} \mathrm{C}$. 


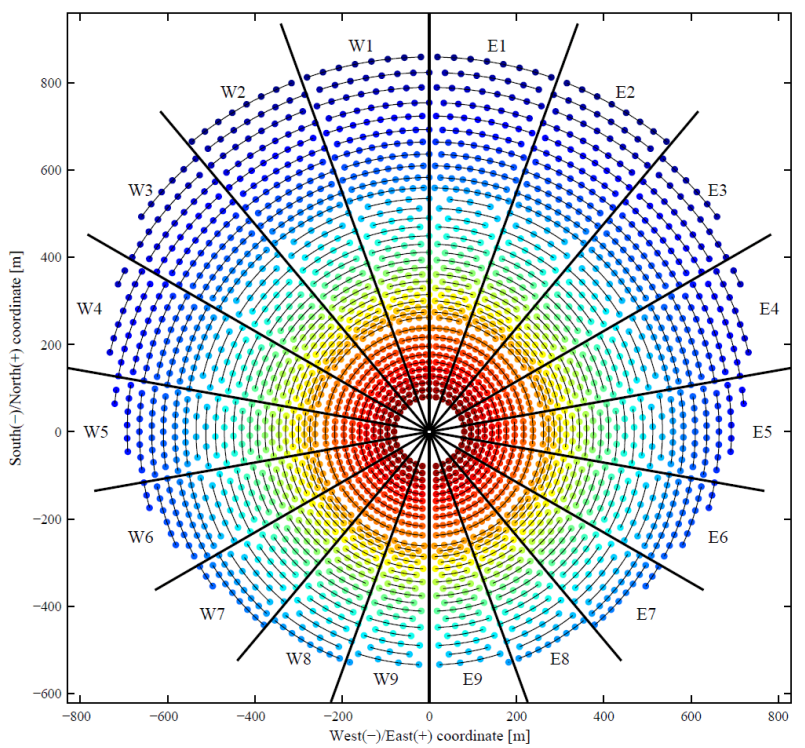

Fig. 13. Layout of Gemasolar heliostat field.

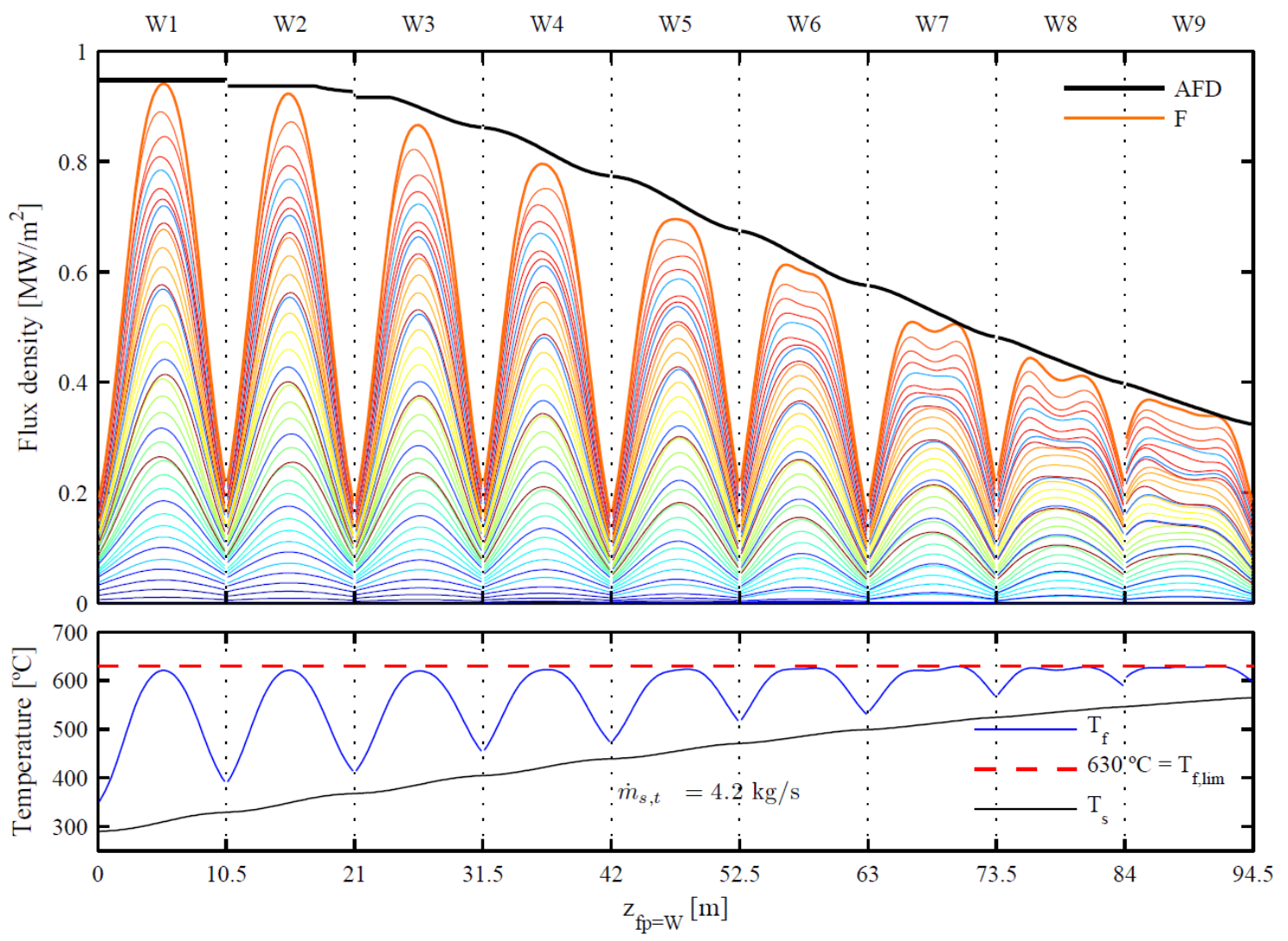

Fig. 14. Fitting at summer solstice noon. Profiles of flux density (top) and temperatures (bottom).

For any instant of time, the aiming strategy model computes the optimal aim points of the field. For example, at 9:00 solar time in summer solstice, Fig. 15 represents the target point of each heliostat according to the color coding in previous Fig.13. Larger shifting is found in the west compared to the less irradiated east side of the receiver. The flux map distribution is shown in the grayscale contours.

Correspondingly to the previous flux distribution at 9:00 summer solstice, Fig. 16 (top) shows the vertical profiles of flux density along the east (left) and the west (right) flowpaths in Gemasolar receiver. The AFD 
limit (red thick line) is not surpassed at any point, so that the thermal stress and the corrosion limit are not broken. The peak flux in each panel is not in the middle, but slightly displaced towards the panel entrance.

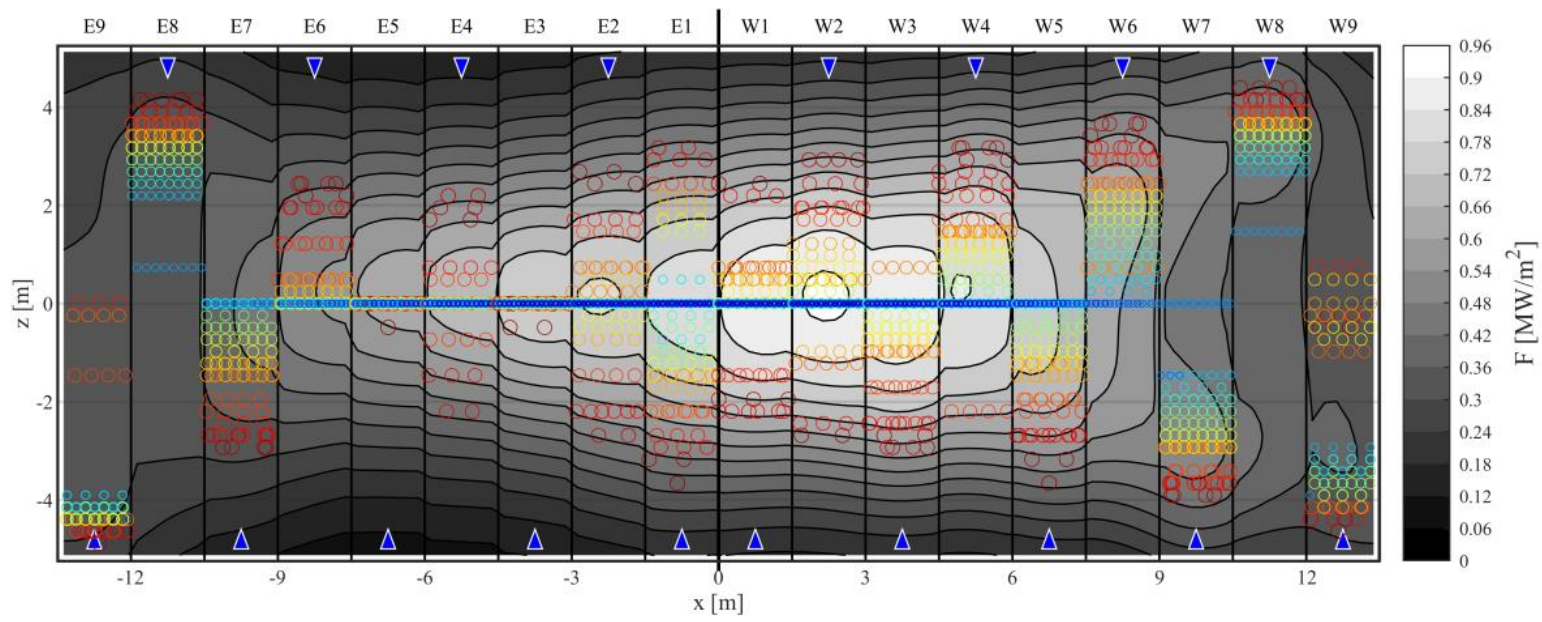

Fig. 15. Flux map at summer solstice $9 \mathrm{~h}$. Circles represent aim points of heliostats according to Fig. 13.
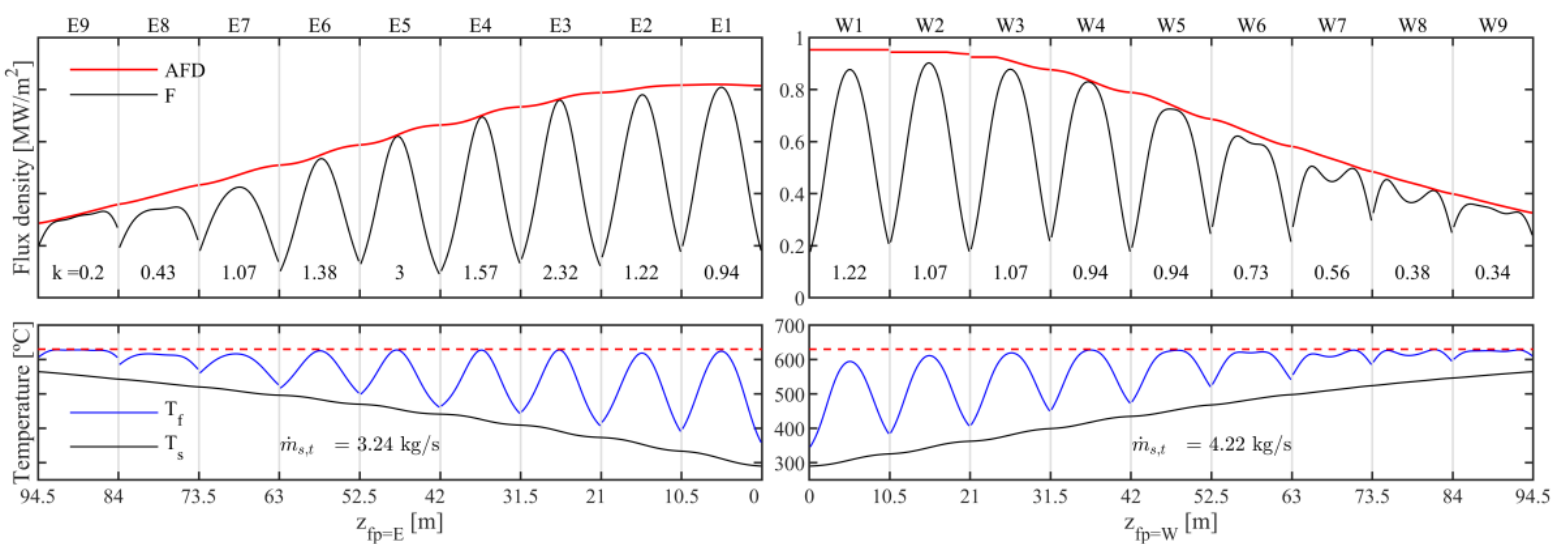

Fig. 16. Flux density (top) and salt and film temperatures (bottom) along east (left) and west (right) flowpaths at summer solstice $9 \mathrm{~h}$.

\section{Conclusions}

Accurate and reliable tools for SPT design and operation are key to guarantee the success and positive attention to this renewable energy technology. In the framework of this doctoral dissertation, four computational models were successfully developed, and experimentally validated, to assist in the design and operation of SPT plants.

The base optical model, on which the rest of the models rely, remarkably reproduced the distorted spot found by rectangular focusing heliostats when the incidence angle on the receiver is large. This is accomplished by the oblique projection of the receiver nodal mesh to the image plane, where the accurate UNIZAR analytic function is used. Compared to Ray Tracing, the model lasts 50 times less; intercept factor is underestimated up to $2 \%$, but spatial resolution is higher in the model.

A novel methodology to determine alignment errors in the facets/mirrors of heliostats was developed. Taking advantage of DIRECT deterministic optimization algorithm, actual canting errors were found by minimizing the difference between experimental and computed flux distributions. For THEMIS heliostats, less than 2-hour computation time is required to find out the 17 angular deviations of the mirror modules, achieving cross-correlation coefficients up to $98 \%$. From the model results, one of the heliostats was in situ 
readjusted, noticeably increasing its optical quality. Compared to the ideal distribution, correlations coefficient rose from $72.8 \%$, before adjusting, to $93.3 \%$; this way validating the proposed methodology.

Two aiming strategies for whole fields of heliostats were also developed. The first one generates symmetric flux maps about the receiver equator, and the resulting software tool, FluxSPT, is freely available. A novel parameter, $k$ aiming factor ranging between 3 (equatorial aiming) and 0 (edge aiming), was defined to estimate the beam radius of the heliostat image on the receiver. In the Dunhuang SPT plant example, $k$ factors to achieve flat flux profiles were found, ranging from 0.6 to 2 depending on the field sector.

The final aiming strategy specifically optimizes the thermal output of molten salt receivers while meeting their structural requirements. Corrosion and thermal stress limitations were translated into allowable flux densities, which can be handled by the optical model. Compared to unreliable single aiming, the optimized aiming strategy increases spillage losses by 4 percentage points, even though the integrity of the receiver is maintained. Because of the serpentine flow pattern in multi-panel cylindrical receivers, it was found that the optimal flux peak is not in the center, but slightly shifted towards the panel entrance.

The code to aim all the heliostats in a field is completely automated. It just takes around 2 minutes on an ordinary PC. Therefore, the aiming strategy model can be applied to real-time control of heliostat fields and molten salt receivers.

\section{Acknowledgements}

The author is indebted to Universidad Carlos III de Madrid for the predoctoral fellowship and the mobility grant that supported this doctoral work between 2013 and 2016. The author appreciates the help and support by: Domingo Santana, thesis advisor; colleague Reyes Rodríguez; Cyril Caliot and Alain Ferriere at PROMES-CNRS laboratory; and Rafael Monterreal at Plataforma Solar de Almería. 